\title{
Organ and Effective Dose Coefficients for Cranial and Caudal Irradiation Geometries: Neutrons
}

\author{
K. G. Veinot ${ }^{1,2, a}$, K.F. Eckerman², N.E. Hertel2,3, M.M. Hiller ${ }^{2}$ \\ 1 Y-12 National Security Complex P.O. Box 2009, M.S. 8206 Oak Ridge, TN, USA 37831 \\ ${ }^{2}$ Center for Radiation Protection Knowledge Oak Ridge National Laboratory Oak Ridge, TN, USA 37831 \\ ${ }^{3}$ Georgia Institute of Technology Atlanta, GA, USA 30332-0745
}

\begin{abstract}
With the introduction of new recommendations by ICRP Publication 103, the methodology for determining the protection quantity, effective dose, has been modified. The modifications include changes to the defined organs and tissues, the associated tissue weighting factors, radiation weighting factors, and the introduction of reference sexspecific computational phantoms (ICRP Publication 110). Computations of equivalent doses in organs and tissues are now performed in both the male and female phantoms and the sex-averaged values used to determine the effective dose. Dose coefficients based on the ICRP 103 recommendations were reported in ICRP Publication 116, the revision of ICRP Publication 74 and ICRU Publication 57. The coefficients were determined for the following irradiation geometries: anterior-posterior (AP), posterior-anterior (PA), right and left lateral (RLAT and LLAT), rotational (ROT), and isotropic (ISO). In this work, the methodology of ICRP Publication 116 was used to compute dose coefficients for neutron irradiation of the body with parallel beams directed upward from below the feet (caudal) and directed downward from above the head (cranial). These geometries may be encountered in the workplace from personnel standing on contaminated surfaces or volumes and from overhead sources. Calculations of organ and tissue absorbed doses for caudal and cranial exposures to neutrons ranging in energy from $10^{-9} \mathrm{MeV}$ to $10 \mathrm{GeV}$ have been performed using the MCNP6 radiation transport code and the adult reference voxel phantoms of ICRP Publication 110. At lower energies the effective dose per particle fluence for cranial and caudal exposures is less than AP orientations while above about $30 \mathrm{MeV}$ the cranial and caudal values are greater.
\end{abstract}

\section{INTRODUCTION}

The International Commission on Radiological Protection (ICRP) Publication 103 [1] established a revised method for determining the protection quantities for organs and tissues (equivalent doses) and the effective dose. Changes to the defined organs and tissues, their associated tissue weighting factors, radiation weighting factors, and the use of sex-specific phantoms from ICRP 110 [2] required a recomputation of dose coefficients. The ICRP DOCAL task group reported these dose coefficients in ICRP Publication 116 [3] which replaces ICRP 74 [4] and the International Commission on Radiation Units and Measurements Report 57 [5]. In ICRP 116 coefficients for the six standard irradiation geometries (anteriorposterior (AP), posterior-anterior (PA), right and left lateral (RLAT and LLAT), rotational (ROT), and isotropic (ISO)) are provided.

Perhaps the most commonly used orientation in the application of the coefficients in practice is the AP geometry since this corresponds to the standard geometry used for personal dosimeter and instrument calibrations. In addition, the ROT irradiation geometry has obvious uses in the computation of effective dose to workers

\footnotetext{
${ }^{\mathrm{a}}$ Corresponding author: veinotkg@y12.doe.gov
}

moving about in a radiation field and the ISO geometry also would have application in practical situations. However, in many operational scenarios these standard geometries may not be appropriate and angular effects, both on the dosimetric quantities as well as the dosimeter response, must be considered. The irradiation geometries of ICRP 116 also do not directly account for the possibility of a person being exposed from sources either beneath the feet (caudal) or from overhead (cranial). In operational settings personnel may perform work on contaminated surfaces or with sources positioned overhead. Since the effective dose is dependent upon the doses delivered to organs and tissues, and because these organs and tissues are shielded differently by the body for different angles of irradion, the caudal (CAU) and cranial (CRA) geometries may result in significantly different effective doses compared to the six standard geometries addressed in ICRP 116.

\section{MATERIALS AND METHODS}

\subsection{Protection Quantity Calculations}


As in previous ICRP recommendations, such as ICRP 26 [6] and ICRP 60 [7], the method for determining protection quantities begins with calculating doses to organs and tissues using mathematical phantoms. In ICRP 103 methodology, the organ and tissue absorbed dose coefficients are determined for the male and female reference voxel phantoms separately, the appropriate radiation weighting factor applied, then the sex-averaged equivalent dose coefficients obtained by averaging the values from the male and female phantoms. The radiation weighting factors, $w_{\mathrm{R}}$, are given in ICRP 103 for various radiations and energies and for neutrons as a continuous function of neutron energy. Once the sex-averaged equivalent doses are determined, they are weighted by the tissue weighting factors and summed to determine the effective dose coefficient. In the ICRP 103 methodology, the specific organs used to define the remainder are prescribed.

ICRP Publication 103 defines effective dose, E, as

$$
E=\sum_{T} w_{T}\left[\frac{H_{T}^{M}+H_{T}^{M}}{2}\right]
$$

where $H_{T}^{M}$ is the equivalent dose to organ $T$ of the male phantom, $H_{T}^{F}$ is the equivalent dose to organ $T$ of the female phantom, and $w_{\mathrm{T}}$ is the organ or tissue weighting factor. The equivalent dose to the organ or tissue is

$$
H_{T}=\sum_{R} w_{R} D_{T, R}
$$

where $w_{\mathrm{R}}$ is the radiation weighting factor and $D_{T, R}$ is the absorbed dose to tissue or organ $T$ from incident radiation $R$.

\subsection{Reference Phantoms}

The reference phantoms of ICRP 110 [2] were used for all calculations presented herein. These three-dimensional phantoms are volumized-pixel (voxel) representations of male and female subjects based on high-resolution scans. The male phantom contains almost two million voxels with each rectangular solid being $8 \mathrm{~mm} \mathrm{X} 2.137 \mathrm{~mm} \mathrm{X}$ $2.137 \mathrm{~mm}$. The female phantom contains almost four million voxels with each being $4.84 \mathrm{~mm} \mathrm{X} 1.775 \mathrm{~mm} \mathrm{X}$ $1.775 \mathrm{~mm}$. Tissue compositions and densities are based on ICRP 89 [8] information. The male phantom is $1.76 \mathrm{~m}$ in height and has a mass of $73 \mathrm{~kg}$ while the female is 1.63 $\mathrm{m}$ tall and has a mass of $60 \mathrm{~kg}$. Both subjects were scanned while laying prone so the phantoms do display some flattening. Additionally, the female head position slightly favors a head down orientation. Because of the voxel resolution, exact calculations to sensitive regions of the skin (e.g. a depth of $0.07 \mathrm{~mm}$ ) and to the lens of the eye are not possible, however, for the calculations reported here the dose to those voxels defining the skin were used. Lens of the eye dose coefficients are not reported. Differences in phantom size and organ mass impact the dose coefficients for certain organs because of shielding effects.

\subsection{Reference Radiation Field Orientations}

For caudal and cranial calculations, planar monoenergetic neutron sources were oriented either below the feet or over the head with particles directed parallel to the longaxis of the phantom. Source areas were sufficiently large to ensure the entire cross-sectional area of the phantom was within the radiation field.

\subsection{Calculational Method}

Calculations were performed using the MCNP6 [9] radiation transport code along with ENDF-B VII cross sections. Both the male and female voxel phantoms from ICRP Publication 110 were modeled and irradiated with planar sources oriented upward from below (CAU) or downward from above (CRA) the phantoms in a vacuum. Organ equivalent doses were computed by tracking the secondary particles via the modified kerma tally $(+\mathrm{F} 6$ in MCNP nomenclature). This provides a more accurate computation of the absorbed dose since it does not assume all energy from interactions is deposited at the interaction site and tracks the secondaries created in the interactions. The dose coefficient energy grid duplicated that used in ICRP Publication 116 and ranged in energy from $10 \mathrm{keV}$ to $10 \mathrm{GeV}$. Organ and effective dose coefficients were normalized to particle fluence and are presented in units of $\mathrm{pSv}-\mathrm{cm}^{2}$.

Because of the segmentation of the cortical (bone surface) and spongiosa (red bone marrow) regions these doses were tallied individually and mass weighted to determine the total bone surface and red bone marrow doses. This method is in accordance with that used for ICRP Publication 116.

Tally uncertainties were minimized, although some organs that received small doses had large tally uncertainties. In cases where these organs and tissues did not significantly contribute to the effective dose this uncertainty was accepted as a practical limitation on computation time. When the organs or tissues were significant contributors to effective dose (the weighted organ equivalent dose, $w_{\mathrm{T}} H_{\mathrm{T}}$, was greater than $1 \%$ of the effective dose), additional particle histories were executed to reduce the uncertainty to less than $3 \%$. In the cases of the bone surface and the red bone marrow the contributor tally errors were propagated using mass weighting. The effective dose error was determined by propagating the sex-averaged, organ weighted equivalent doses in quadrature.

\section{RESULTS AND DISCUSSION}

Effective dose coefficients for cranial (CRA) and caudal (CAU) exposures are shown in Fig. 1 along with AP irradiation geometry values from ICRP Publication 116. Ratios of the CAU and CRA effective dose coefficients to AP are shown in Fig. 2. Tabulated values of the effective dose and all organs and tissue equivalent dose coefficients are available for download from the Oak 
Ridge National Laboratory Center for Radiation Protection Knowledge website (http://crpk.ornl.gov/resources/) or can be obtained by contacting the authors.

The effects of caudal and cranial exposures on effective dose are evident, and not unexpected. Differences in organ doses between the male and female were noted and are a consequence of organ location within the body (effects of different body shielding of internal organs for different angles of incidence), phantom mass and size, and phantom posture itself. For example, the gonads and brain dose are two organs that exhibit the highest dependence on irradiation orientation. Ratios of the organ equivalent dose for the two geometries for the brain and gonads are shown in Fig. 2.

\section{CONCLUSION}

Dose coefficients for caudal and cranial irradiation geometries have been calculated in accordance with ICRP Publication 103. The coefficients differ significantly from AP values for both organ and tissues of ICRP Publication 116, as would be expected. The differences can lead to significant overestimation of personnel doses if the AP irradiation geometry is assumed.

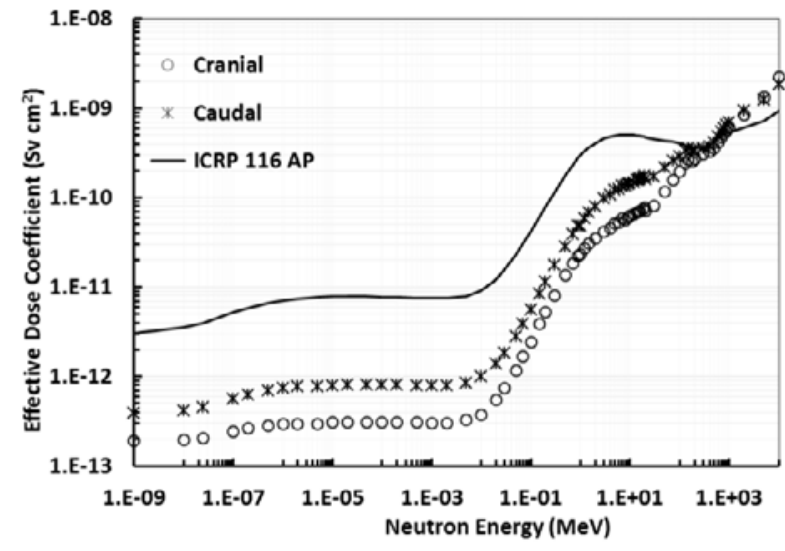

Figure 1. Effective dose coefficients for cranial and caudal irradiations and for AP as listed in ICRP 116.

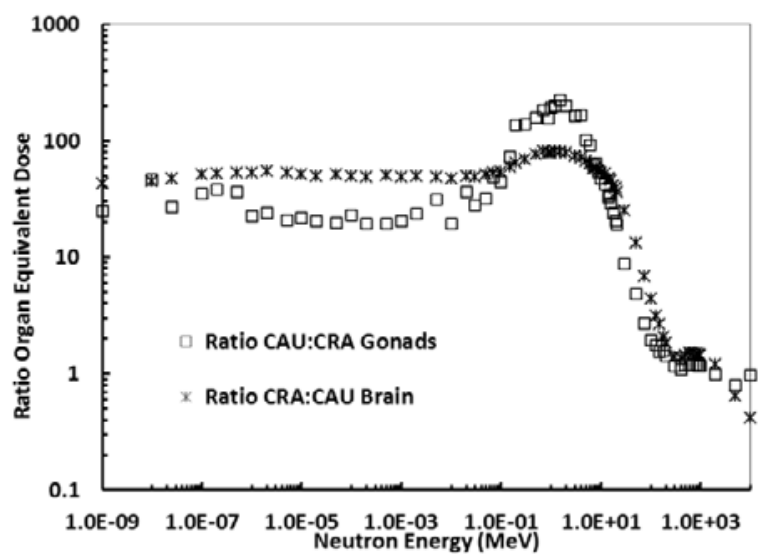

Figure 2. Ratio of organ equivalent doses for cranial and caudal geometries for the brain and gonads..

\section{References}

1. INTERNATIONAL COMMISSION ON RADIOLOGICAL PROTECTION (ICRP), "The 2007 Recommendations of the International Commission on Radiological Protection," ICRP Publication 103. Ann. ICRP 37 (2-4). Elsevier (2007).

2. INTERNATIONAL COMMISSION ON RADIOLOGICAL PROTECTION (ICRP), "Adult Reference Computational Phantoms," ICRP Publication 110. Ann. ICRP 39 (2). Elsevier (2009).

3. INTERNATIONAL COMMISSION ON RADIOLOGICAL PROTECTION (ICRP), "Conversion Coefficients for Radiological Protection Quantities for External Radiation Exposures," ICRP Publication 116. Ann. ICRP 40 (2-5). Elsevier (2010).

4. INTERNATIONAL COMMISSION ON RADIOLOGICAL PROTECTION (ICRP), "Conversion Coefficients for Use in Radiological Protection Against External Radiation," ICRP Publication 74. Ann. ICRP 26 (3-4). Pergamon Press (1996).

5. INTERNATIONAL COMMISSION ON RADIATION UNITS AND MEASUREMENTS (ICRU), "Conversion Coefficients For Use In Radiological Protection Against External Radiation," ICRU Report 57, Bethesda, MD; ICRU (1998).

6. INTERNATIONAL COMMISSION ON RADIOLOGICAL PROTECTION (ICRP), "Recommendations of the International Commission on Radiological Protection," ICRP Publication 26. Ann. ICRP 1 (3). Pergamon Press (1977).

7. INTERNATIONAL COMMISSION ON RADIOLOGICAL PROTECTION (ICRP), "1990 Recommendations of the International Commission on Radiological Protection," ICRP Publication 60. Ann. ICRP 21 (1-3). Pergamon Press (1991).

8. INTERNATIONAL COMMISSION ON RADIOLOGICAL PROTECTION (ICRP), "Basic Anatomical and Physiological Data for Use in Radiological Protection Reference Values," ICRP Publication 89. Ann. ICRP 32 (3-4). Pergamon Press (2002).

9. MCNP6 USER'S MANUAL Version 1.0, Denise B. Pelowitz, Ed., LA-CP-13-00634 Rev. 0, Los Alamos National Laboratory Los Alamos, New Mexico (2013). 